\title{
TREATMENT OF ACUTE ANKLE SYNDESMOTIC INJURY BY SYNDESMOTICS SCREWS FIXATION
}

\author{
By
Ismail Hammoudah, Faisal Hasan Zayed and Mohamed Abd El-Naser Haggag

Department of Orthopedic Surgery, Faculty of Medicine, Al-Azhar University

Corresponding author: Mohamed Abdelnaser Haggag,

Mobile: 01000895840; E-mail: dr.mido136@gmail.com

\begin{abstract}
Background: The distal tibiofibular syndesmosis is a connection between tibia and fibula that consists of the anterior tibiofibular ligament, the posterior tibiofibular ligament, the transverse ligament and the interosseous ligament. Syndesmotic ligament injuries are most often seen in combination with ankle fractures, but can occur in isolation as well. The term 'isolated syndesmotic injury' is used for the syndesmotic rupture without ankle fracture, although concomitant other injuries may be present. These isolated injuries, which are often accompanied by deltoid ligament injuries.
\end{abstract}

Objective: To evaluate the efficiency of use of syndesmotic screw fixation in treatment of acute ankle syndesmotic injury.

Patients and Methods: This Prospective study included twenty adult patients with recent ankle syndesmotic injury who were treated by syndesmotic screw at Al-Azhar university hospital and mansheyat El-bakry hospital, Cairo, Egypt. Those twenty patients prospectively followed up for 12 months with average of 9 months. The clinical outcomes were calculated according AOFAS score.

Results: Those 20 patients prospectively followed up for an average of 9 months. Preoperative mean value for medial clear space (MCS) was $11.8 \pm 3.9 \mathrm{~mm}$, tibiofibular clear space (TFCS) was $9.2 \pm 1.6 \mathrm{~mm}$ and tibiofibular overlap (TFO) was $2.2 \pm 4.7 \mathrm{~mm}$. while the follow up mean MCS was $3.3 \pm 0.4 \mathrm{~mm}$, TFCS was $3.2 \pm 0.4 \mathrm{~mm}$ and TFO was measured as $11.9 \pm 1.2 \mathrm{~mm}$ The mean AOFAS score was 97.0 \pm 3.8 (range 90-100).

Conclusion: Reduction of the syndesmosis is essential for improving functional outcomes and avoiding posttraumatic osteoarthritis. It is important to intraoperatively stress all surgically treated ankle fractures to evaluate latent syndesmotic injury.

Keywords: Distal tibio-fibular syndesmosis, Anterior inferior tibiofibular ligament, Range of motion.

\section{INTRODUCTION}

Ankle fractures are common injuries treated by orthopaedic surgeons. These injuries are increasing in number due to a more active, aging population. Ankle fractures where the ankle mortise is stable and there is adequate alignment are usually treated nonoperatively. Stability and reduction quality can be determined simply in the coronal plane, but syndesmotic instability and posterior malleolar stability are not as easily established and has been the subject of much research and debate (Ovaska et al., 2013).

Previously, syndesmotic instability has been thought to be predicted based on Lauge-Hansen classification and fibular fracture height. More recent studies have shown that syndesmotic disruption can 
happen with almost any fracture pattern. The intraoperative stress examination of the syndesmosis is currently the gold standard for determining the need for stabilization, although a preoperative assessment with computed tomography (CT) and magnetic resonance imaging (MRI) may be useful in predicting syndesmotic disruption (Egol et al., 2010).

Biomechanical studies suggest that fixation of the posterior malleolus will restore the posterior aspect of the tibiofibular ligament, obviating the need for syndesmotic stabilization. These data have yet to be shown in a large clinical series. The screw fixation method is the conventional treatment in syndesmosis repair. Rigid fixation is performed via the passage of a cortical screw through the fibula at a level of $2 \mathrm{~cm}$ superior to the tibiotalar joint and parallel to the joint, into the tibia and 300anteriorly (Nousiainen et al., 2011).

The syndesmosis screw should be removed 3 months after the procedure. If the screw is not removed and remains unbroken, local symptoms may be developed (Manjoo et al., 2010).

Recently, elastic fixation materials have been used, which are alternatives to screws. Despite the doubts concerning their biomechanical adequacy, they have some advantages over the rigid fixation method as the need for removal of the implant is less, and they possess a more dynamic and physiological property compared to screw fixation (Coetzee and Ebeling, 2011).

The aim of the study was to evaluate the efficiency of use of syndesmotic screw fixation in treatment of acute ankle syndesmotic injury.

\section{PATIENTS AND METHODS}

This Prospective study included twenty adult patients with recent ankle syndesmotic injury who were treated by syndesmotic screw at Al-Azhar University Hospital and Mansheyat El-Bakry Hospital, Cairo, Egypt.

Those patients were prospectively followed up for 12 months with average of 9 months. The clinical outcomes were calculated according to AOFAS score.

The study was approved by the ethics committee of our institute.

\section{Inclusion criteria:}

Skeletally mature patient. Syndesmotic injury with or without ankle fracture. Syndesmotic injury with fracture dislocation ankle.

\section{Exclusion criteria:}

Pathological fracture. Open fracture. Skeletally immature. Neuropathic joint. Pilon fracture.

All patients were submitted for oral and written consents.

Preoperative evaluation: The patients were managed according to advanced trauma life support principles in the first instance. Assessment of the neurovascular status of the limb was mandatory. The patients were examined clinically to evaluate the soft tissue condition, and Xrays were obtained in the anteropsterior, lateral and mortise views. Patients were put in a back slab until time of surgery. CT has been done in only one case. Metal screws were traditionally used for syndesmotic fixation, but there is considerable variability in screw selection. Fifteen cases were fixed by one screws 
three cortices except two cases were fixed by two screws four cortices and three cases were fixed by one screw four cortices.

\section{Post-operative}

management:

Administration of IV antibiotics for 3 days postoperative. Hospital stay ranged between 2-3 days post-operative. Postoperative slab was applied. A commonly used post-operative regimen includes no weight bearing until screw removal, with an average of 6-11 weeks, to allow enough time for healing of the disrupted syndesmotic ligaments. Recurrence of syndesmotic diastasis following early screw removal has been described; alternatively, weight bearing can be initiated with syndesmotic screw(s) in place. In such instances, the patient should be warned that the screw loosening and/or breakage may occur, prefer to keep the patient non-weight bearing for 6 weeks after which the patient begins weight bearing in a short leg walking cast for 2 weeks followed by the use of a soft ankle brace for 4 weeks.

Clinical and radiological follow up were scheduled at 2 week, 4 weeks, and 2 months post operatively. All screws were removed in the operating room under local anesthesia after a time from 6weeks up to 8 weeks.

All patients were followed up by X-ray $\mathrm{AP}$ and lateral view and clinical evaluation of pain and tenderness.

\section{Statistical analysis:}

The data were coded and entered using the statistical package SPSS version 15 . The data were summarized using descriptive statistics: mean, standard deviation, maximal and minimal values for quantitative variables and number and percentage for qualitative values. $\mathrm{P}$ values less than or equal 0.05 were considered statistically significant.

\section{RESULTS}

In this prospective study, twenty adult patients with recent ankle syndesmotic injury were treated with syndesmotic screw fixation. Those 20 patients prospectively followed up for an average of 9 months. Preoperative mean value for medial clear space (MCS) was 11.8 \pm 3.9 $\mathrm{mm}$, tibiofibular clear space (TFCS) was $9.2 \pm 1.6 \mathrm{~mm}$ and tibiofibular overlap (TFO) was $2.2 \pm 4.7 \mathrm{~mm}$. while the follow up mean MCS was $3.3 \pm 0.4 \mathrm{~mm}$, TFCS was $3.2 \pm 0.4 \mathrm{~mm}$ and TFO was measured as $11.9 \pm 1.2 \mathrm{~mm}$ The mean AOFAS score was $97.0 \pm 3.8$ (range 90-100).

From 20 patients, 19 underwent removal of syndesmotic screw after 68 weeks, and 1 underwent removal of plates because of prominence hardware and irritation.

There was a statistical significant difference ( $p$-value < 0.001 ) between preoperative and post-operative MCS, TFCS and TFO in studied patients (Table 1). 
Table (1): Comparison pre-operative and post-operative MCS, TFCS and TFO in studied patients

\begin{tabular}{|c|c|c|c|c|c|}
\hline \multirow{2}{*}{ Groups } & \multicolumn{2}{|c|}{$\begin{array}{c}\text { Pre-operative } \\
\mathbf{N}=(\mathbf{2 0})\end{array}$} & \multicolumn{2}{c|}{$\begin{array}{c}\text { Post-operative } \\
\mathbf{N}=(\mathbf{2 0})\end{array}$} & t-test \\
\cline { 2 - 6 } & Mean & $\mathbf{\pm S D}$ & Mean & $\mathbf{\pm S D}$ & p-value \\
\hline MCS (mm) & 11.8 & 3.9 & 3.3 & 0.4 & $<\mathbf{0 . 0 0 1}$ \\
\hline TFCS (mm) & 9.2 & 1.6 & 3.2 & 0.4 & $<\mathbf{0 . 0 0 1}$ \\
\hline TFO (mm) & 2.2 & 4.7 & 11.9 & 1.2 & $<\mathbf{0 . 0 0 1}$ \\
\hline
\end{tabular}

There was a statistical significant difference $(\mathrm{p}$-value $<0.001)$ between
AOFAS follow up in studied patients (Table 2).

Table (1): Comparison between AOFAS follow up in studied patients

\begin{tabular}{|c|c|c|c|c|c|c|c|}
\hline \multirow{2}{*}{ Parameters Groups } & \multicolumn{2}{|c|}{$\begin{array}{l}6 \text { weeks } \\
N=(20)\end{array}$} & \multicolumn{2}{|c|}{$\begin{array}{c}3 \text { Months } \\
\mathbf{N}=(20)\end{array}$} & \multicolumn{2}{|c|}{$\begin{array}{c}6 \text { Months } \\
\mathbf{N}=(20)\end{array}$} & \multirow{2}{*}{$\begin{array}{l}\text { ANOVA } \\
\text { p-value }\end{array}$} \\
\hline & Mean & \pm SD & Mean & \pm SD & Mean & \pm SD & \\
\hline AOFAS & 64.5 & 7.5 & 86.1 & 2.4 & 97.0 & 3.8 & $<0.001$ \\
\hline
\end{tabular}

\section{DISCUSSION}

The result of this study was that syndesmotic screw effective in treatment of syndesmotic injury with good postoperative results $(80 \%)$ and some complications as moderate pain, hardware irritation and limitation of range of motion $(20 \%)$.

Schepers et al. (2014) reported that the functional outcome of acute syndesmotic injuries treated with a syndesmotic screw was good and mainly influenced by patient and fracture characteristics. Most different technical aspects of placement appeared not to influence these results. Only screw placement above $41 \mathrm{~mm}$ negatively influenced outcome.

Schon et al. (2016) reported that syndesmotic screw has good results in treatment of syndesmotic injury as suture button in post-operative diastasis.this study not used suture button but used syndesmotic screw with good result and some complications.
Van der Eng et al. (2015) in a grade I evidence research reported that syndesmotic screws have good postoperative results with less complications than bio-absorbable screws. This research studied 260 cases. The experimental group consisted of patients with syndesmotic injuries treated with bioabsorbable screws versus the control group (patients treated with metallic screws). The most common complications were wound infection and decreased range of motion. In this study all cases were treated with metallic screw not bioabsorbable screw with good results and some complications.

Kortekangas et al. (2015) reported that there is a degree of mal reduction after two years of syndesmotic screw removal, but the study depended only on CT study without clinical correlation. This study depended on $\mathrm{x}$-ray and clinical evaluation not on CT with good result.

Grassi et al. (2019) reported that there is high rate of two years post-operative mal-reduction in syndesmotic screw 
fixation, so it's better to use dynamic stabilization in treatment of syndesmotic injury in this study there was no malreduction in syndesmotic screw.

Shimozono et al. (2018) reported that there are more complications in syndesmotic screws fixation than suture button. In this study the complications were limited as moderate pain, hardware irritation, infection, and limitation of range of motion.

Bafna et al. (2019) reported that the complications of syndesmotic screws may be recurrent diastasis, broken screws, screw loosening, osteomyelitis, osteoarthritis or fibular nonunion in this study these complications not reported.

Zhang et al. (2017) reported that postoperative results of syndesmotic screws are good with low complication rate as suture button. This study reported that good results with syndesmotic screw.

Michelson et al. (2018) reported that the number or placement of syndesmotic screws or the breakage of transsyndesmotic screws postoperatively had no adverse effect on outcomes (both with moderate strength of evidence). The use of alternative fixation devices (bioabsorbable and end button) had poor strength of evidence, as did the opinion that nondisplaced, unstable by stress test, syndesmotic injuries required fixation. There are insufficient data that link subtle rotational syndesmotic mal reduction to clinical outcomes.in this study the fixation was by metallic screw not bioabsorbable nor button with good results.

Patel et al. (2018) reported that tricortical syndesmotic screw increases lateral talar shift which accelerate ankle arthritis, so syndesmotic screw must be removed as early as possible.in this study the fixation was by tricortical and quadricortical syndesmotic screw with good results and little complications.

Clanton et al. (2017) reported that all methods of surgical fixation provided comparable rotational stability to the syndesmosis; however, no repair technique completely restored rotational stability and tibiofibular anatomic relationships of the preinjury state.

Murphy et al. (2017) reported that tricortical screw restored fibular motion in all planes. No significant differences were observed compared to the intact ankle.in this study tricortical and quadricortical syndesmotic screw restored fibular motion in all planes.

Xenos et al. (2012) found a higher mechanical strength in two-screw fixation compared with one screw fixation. In a randomised study in which one- and twoscrew fixation was compared, no differences were found in functional outcome, dorsiflexion and pain. Suggestions for using two-screw fixation in obese patients, non-compliant patients or in case of complete diastasis have been made. In this study $18(90 \%)$ of patients were fixed by one screw and $2(10 \%)$ of patients were fixed by two screws, no differences were found in functional outcome, dorsiflexion and pain.

\section{CONCLUSION}

Anatomic reduction of the syndesmosis is essential for improving functional outcomes and avoiding posttraumatic osteoarthritis. It is important to intraoperatively stress all surgically 
ISMAIL HAMMOUDAH et al.,

treated ankle fractures to evaluate latent syndesmotic injury.

\section{REFERENCES}

1. Bafna KR, Jordan R, Yatsonsky D, Dick S, Liu $J$ and Ebraheim NA (2019): Revision of Syndesmosis Screw Fixation. Foot \& Ankle Specialist, 1(2):1-8.

2. Clanton TO, Whitlow SR, Williams BT, Liechti DJ, Backus JD and Dornan GJ (2017): Biomechanical comparison of 3 current ankle syndesmosis repair techniques. Foot \& Ankle International, 38(2):200-7.

3. Coetzee JK and Ebeling PB (2011): Treatment of syndesmoses disruptions: a prospective, randomized study comparing conventional screw fixation vs TightRope1 fiber wire fixation-medium term results. SA Orthop J Autumn, 32-7.

4. Egol KA, Pahk B, Walsh M, Tejwani NC, Davidovitch RI and Koval KJ (2010): Outcome after unstable ankle fracture: effect of syndesmotic stabilization. J Orthop Trauma, 24(1):7-11.

5. Grassi A, Samuelsson K, D'Hooghe P, Romagnoli M, Mosca M and Zaffagnini S (2019): Dynamic Stabilization of Syndesmosis Injuries Reduces Complications and Reoperations as Compared With Screw Fixation: A Meta-analysis of Randomized Controlled Trials. The American Journal of Sports Medicine, 2(5): 1-14.

6. Kortekangas $T$, Savola O, Flinkkilä $T$, Lepojärvi $S$, Nortunen $S$ and Ohtonen $P$ (2015): A prospective randomised study comparing TightRope and syndesmotic screw fixation for accuracy and maintenance of syndesmotic reduction assessed with bilateral computed tomography. Injury, 46(6):111926.

7. Manjoo A, Sanders DW, Tieszer $\mathrm{C}$ and MacLeod MD (2010): Functional and radiographic results of patients with syndesmotic screw fixation: implications for screw removal. J Orthop Trauma, 24(1):2-6.

8. Marmor M, Hansen E, Han HK, Buckley J and Matityahu A (2011): Limitations of standard fluoroscopy in detecting rotational malreduction of the syndesmosis in an ankle fracture model. Foot \& Ankle International, 32(6):616-22.

9. Michelson JD, Wright $M$ and Blankstein M (2018): Syndesmotic ankle fractures. Journal of Orthopaedic Trauma, 32(1):10-4.

10. Murphy C, Pfeiffer T, Zlotnicki J, Musahl V, Debski $R$ and Hogan $M$ (2017): Restoration of Tibiofibular Kinematics with a Tricortical Screw or Suture Button Fixation After Syndesmotic Ankle Injuries. Foot \& Ankle Orthopaedics, 2(3):1-10.

11. Nousiainen MT, McConnell AJ, Zdero R, McKee MD, Bhandari $M$ and Schemitsch EH (2011): The influence of the number of cortices of screw purchase and ankle position in Weber $\mathrm{C}$ ankle fracture fixation. J Orthop Trauma, 22(7):473-8.

12. Nussbaum ED, Hosea TM, Sieler SD, Incremona BR and Kessler DE (2010): Prospective evaluation of syndesmotic ankle sprains without diastasis. The American Journal of Sports Medicine, 29(1):31-5.

13. Ovaska MT, Ma"kinen TJ, Madanat R, Vahlberg T, Hirvensalo $\mathbf{E}$ and Lindahl $\mathrm{J}$ (2013): Predictors of poor outcomes following deep infection after internal fixation of ankle fractures. Injury, 44(7):1002-6.

14. Patel NK, Pfeiffer TR, Naendrup J-H, Murphy C, Zlotnicki $J$ and Debski $R$ (2018): Talar Motion is Constrained by Tricortical Screw Fixation of the Syndesmosis: A Cadaveric Robotic Study. Orthopaedic Journal of Sports Medicine, 6(7): 2-18

15. Schepers $T$, van der Linden $H$, van Lieshout EM, Niesten DD and van der Elst M (2014): Technical aspects of the syndesmotic screw and their effect on functional outcome following acute distal tibiofibular syndesmosis injury. Injury, 45(4):775-9.

16. Schon JM, Williams BT, Venderley MB, Backus JD, Dornan GJ and Turnbull TL (2016): 3-D CT Analysis of Screw and Suture-Button Fixation for Syndesmosis 
Repair. Foot \& Ankle Orthopaedics, 1(1): 510.

17. Shimozono Y, Hurley ET, Myerson CL, Murawski CD and Kennedy JG (2018): Suture Button Versus Syndesmotic Screw for Syndesmosis Injuries: A Meta-analysis of Randomized Controlled Trials. The American Journal of Sports Medicine, 47(11):2764-71.

18. Van der Eng DM, Schep NW and Schepers T (2015): Bioabsorbable versus metallic screw fixation for tibiofibular syndesmotic ruptures: a meta-analysis. The Journal of Foot and Ankle Surgery, 54(4):657-62.
19. Xenos JS, Hopkinson WJ and Mulligan ME (2012): The tibiofibular syndesmosis. Evaluation of the ligamentous structures, methods of fixation, and radiographic assessment. Journal of Bone and Joint Surgery, 77-A: 847-56.

20. Zhang $P$, Liang $Y$, He J, Fang $Y$, Chen $P$ and Wang $J$ (2017): A systematic review of suture-button versus syndesmotic screw in the treatment of distal tibiofibular syndesmosis injury. BMC Musculoskeletal Disorders, 18(1):286-290. 


\section{علاج القطع الحاد بالرباط الأحادى للكاحل بواسطة مسمار إسماعيل حموده، فيصل حسن زايد، محمد عبد الناصر حجاج}

قسم جراحة العظام، كلية الطب، جامعة الأزهر

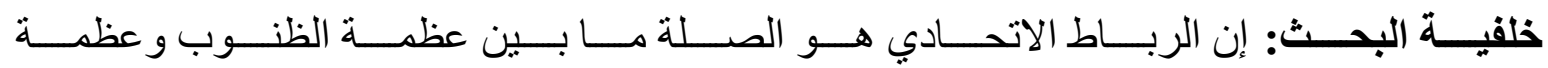

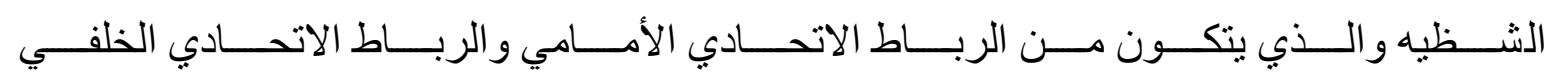

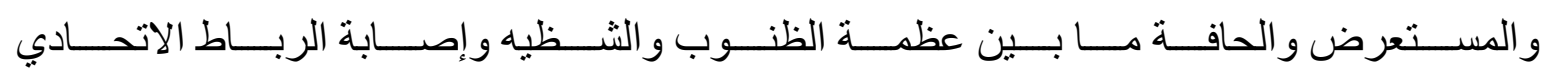

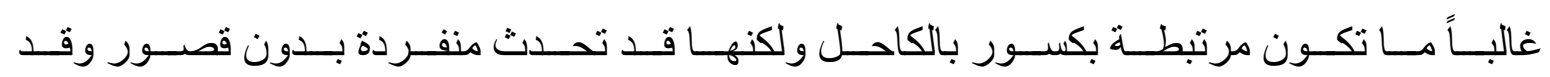
يصاحب ذلك تمزق بالرباط الدالي للكاحل.

الهُف من البحث: تقييم كفاءة تتبيت القطع الحاد برباط الأتحادي بو اسطة مسمار.

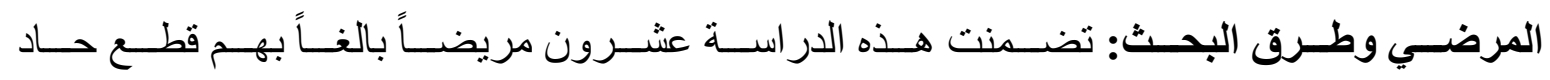

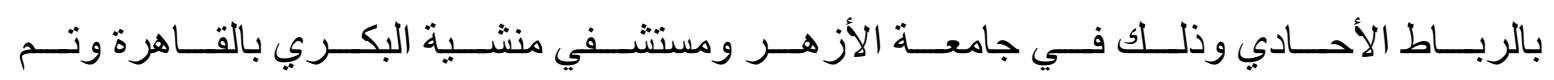

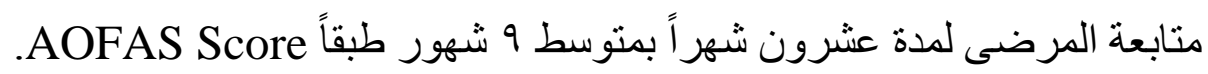

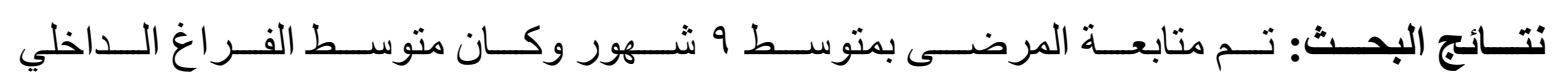

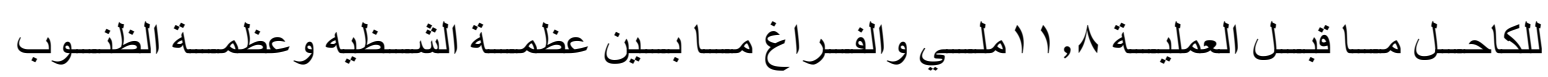

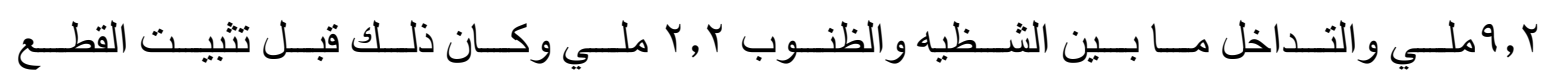

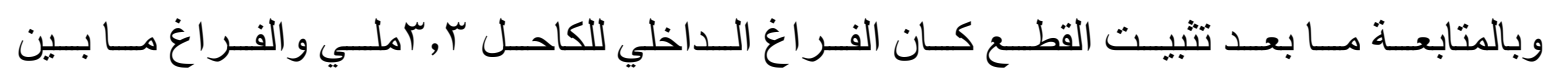

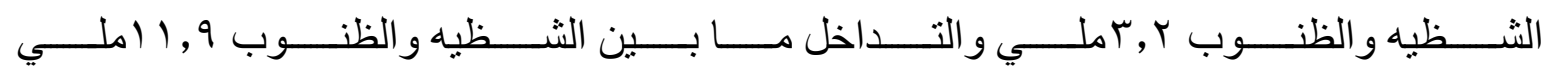
و المتوسط

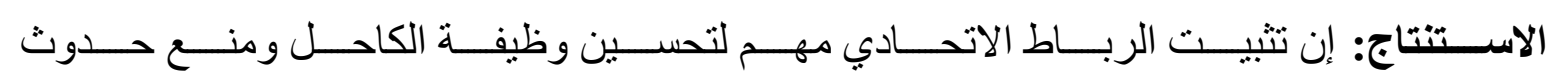

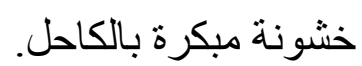

